\title{
Use of Modelica to predict risk of Covid-19 infection in indoor environments
}

\author{
Arnav Pathak Kilian Schneider Victor Norrefeldt \\ Fraunhofer-Institute for Building Physics, Fraunhoferstr. 10, D-83626 Valley, Germany \\ victor.norrefeldt@ibp.fraunhofer.de
}

\begin{abstract}
In the light of the Sars-CoV-2 pandemic, the dispersion process of respiratory droplets released by potentially infected persons has been investigated in many studies using highly reliable but time consuming CFD methods. With such simulations social distancing, wearing masks and shifts in ventilation systems could be justified. This work focuses on the same topic but uses the validated Velocity Propagating Zonal Model (VEPZO) instead of CFD simulations. It is implemented in Modelica and allows fast simulation of the indoor environment on a coarse grid which in many cases is a superior alternative to complex CFD simulations in the trade-off between effort and detail of the result. Based on the temperature and airflow distribution, this model can be used to predict the dispersion of aerosols in enclosed spaces and thus the relative risk of Covid-19 infection. For model verification, a documented outbreak in a restaurant in Guangzhou is being investigated. An improved ventilation pattern to contain viral load more locally is developed.
\end{abstract}

\section{Introduction}

Covid-19 is mainly transmitted from human-to-human through inhalation of respiratory droplets. Transmission of respiratory infectious diseases has been studied in different disciplines for decades. Computational Fluid Dynamics (CFD) simulation is an accurate and reliable method to predict airflow patterns and thus lately the risk of airborne cross infection in a crowded indoor environment with an infected index patient. Zhang and Li (2012) studied the dispersion process of respiratory droplets released by a coughing person in a high speed rail cabin using CFD simulations. Four cases of different air supply and exhaust locations are reviewed. The droplets' dispersion characteristics and the maximum dispersion distances under specified ventilation conditions are investigated. This study demonstrates the potential of improving cabin air ventilation for infection control. In Goscé et al. (2014) the authors show the dependency of walking speed on the rate of infection in crowded underground corridors. Recent discussions investigated infection risks also in aircraft cabins and other indoor environments such as offices, classrooms and restaurants. Yan et al. (2017) simulated a whole fully occupied 7 row aircraft cabin and investigated the influence of different ventilation systems on the droplet dispersion for different respiratory activities of a single person. The key particle transport information such as the particle residence time yielded from the Lagrangian tracking process was extracted and integrated into the Wells-Riley equation (Sze To and Chao 2010) in conjunction with CFD predictions. Villafruela et al. (2016) focus on the validation of a 3-D transient CFD model used to predict personal exposure to airborne pathogens and infection risk in a displacement ventilated room. After consideration of different interactions of two exhaling persons, they conclude that numerical simulations have the capacity to analyse the dispersion of exhaled contaminants over time.

Melikov (2020) postulated a paradigm shift in ventilation design which is needed in order to respond to the current need. State of the art ventilation is mainly based on mixing air distribution. This does neither provide efficient removal of polluted air exhaled by occupants before mixing nor the direct supply of clean air to the breathing zone.

The investigated case of this paper is a so called super spreader event taken place in a restaurant in Guangzhou, China (Lu et al. 2020). With the help of the Velocity Propagating Zonal model (VEPZO) (Norrefeldt et al. 2012) the case could be reconstructed and the infection spread traced back to a poorly ventilated dining room. Furthermore an alternative to the existing ventilation system is developed in order to contain the spread of potentially infectious aerosols.

\section{Methods}

\subsection{Implementation of the VEPZO model}

Zonal models allow simpler and faster estimation of indoor climate than complex CFD simulations. They use similar mathematical theory, but subdivide the space more coarsely into 10 to 100 zones exchanging air through flow paths. These are usually based on the Bernoulli-equation, causing numerical issues and a lack of precision at a zero pressure difference due to the square root function. The Velocity Propagating Zonal Model (VEPZO) is an advanced type of zonal model, developed at the Fraunhofer-Institute for Building Physics. By introducing airflow velocity vectors and making them a property of the zone, the airflow velocity 
is no longer dissipated once it has entered a zone but propagates into space. Therefore the zonal formulation is still valid in areas with driving flows due to jets or plumes and overcomes the need for correlation formulation in those areas.

The two main components of the VEPZO model are a zone model and a flow model (Figure 1). The zone (cube) and the flow (grey rectangle) models are connected by ports (rhombs) to form a room. These ports allow the exchange of relevant information between the flow and the zone model. The flow models have two ports to connect adjacent zones. Each zone has six ports, one for each boundary. A Boolean parameter is assigned to each port to make the distinction whether the port is connected to a flow model or whether there is no flow because the zone is adjacent to a room boundary surface.

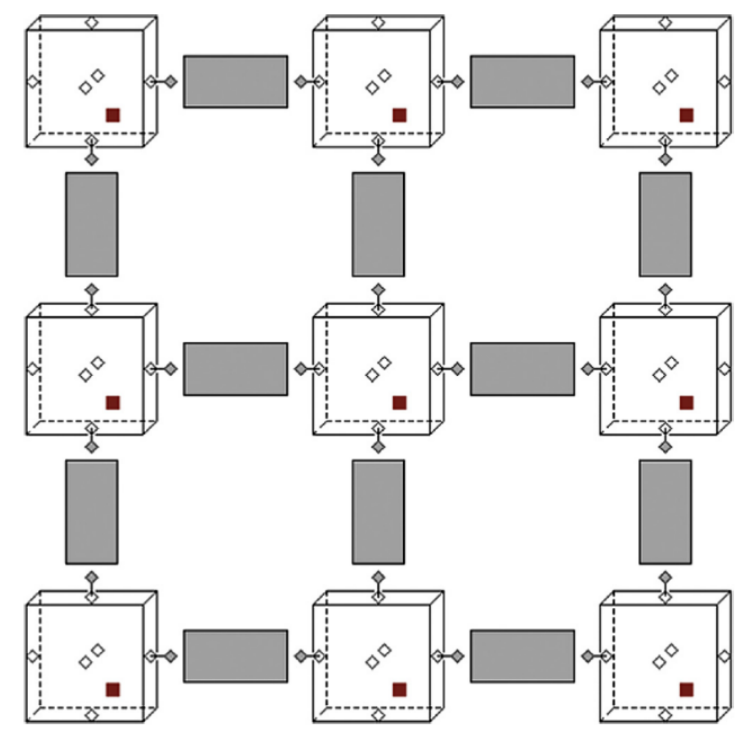

Figure 1: Zonal model in $x-z$ direction (y not shown); cubes: zones; grey rectangles: flows; rhombs: airflow ports; red solid squares: heat ports.

Each zone has a heat port (red square) allowing exchanges with models of other components like e.g. heat sources or walls to assess the local impact of these heat releases on the airflow pattern and the temperature distribution in a room. Models computing the interaction of air properties like density, pressure, temperature or specific enthalpy are available in the Modelica.Media library and are used in the VEPZO model (Figure 2). Depending on the application, the air model can be changed from dry to moist air. Pollutants can be taken into account by adding a tracer substance (ExtraProperty) to the Media model.

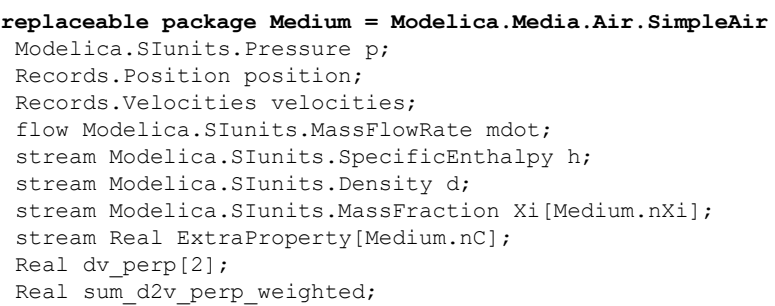

Figure 2: Implementation of connector, position and velocities

\subsection{Zonal Model}

The main task of the zone model is to compute the mass and enthalpy balance and air properties (density, enthalpy, pressure, temperature, etc.) using air models of Modelica.Media. Furthermore it determines a characteristic velocity and viscous losses.

The zone model contains interfaces to various types of fluxes, including those of air, gases and particles.

Air contained in a zone is assumed to be perfectly mixed. The mass conservation takes into account the amount of air exchanged with adjacent zones and airflows provided by various sources or sinks (ventilation, openings, etc.) in the zone. Heat flows due to convection to walls or heat sources contained in the zone are added to the thermal energy balance. A new feature of the VEPZO model is that a characteristic velocity vector $(\mathrm{u}, \mathrm{v}, \mathrm{w})$ is assigned to the zones. Knowing the mass flow and its direction across each of the zone's surfaces, the flow velocity across these surfaces is determined. The zone shares the information about its characteristic velocity with the flow models surrounding it. This enables the VEPZO model to propagate the airflow velocity throughout the room without needing special correlations like jets or plumes.

\subsection{Flow model}

The main task of the flow model is to compute the airflow rate between two adjacent zones. Furthermore, the flow models are used to calculate the velocity gradient needed for the calculation of viscous losses. Two adjacent zones are connected by a flow model computing the exchange of air between them. The VEPZO model uses flow models in $x^{-}, y-$ and $z$ directions. The assumption of the VEPZO model is that air only flows along these specific directions. A new feature of the flow model used in the VEPZO model is that the length of a flow path is taken into account. The flow model computes the airflow acceleration or deceleration from the forces acting on it (Figure 3). 


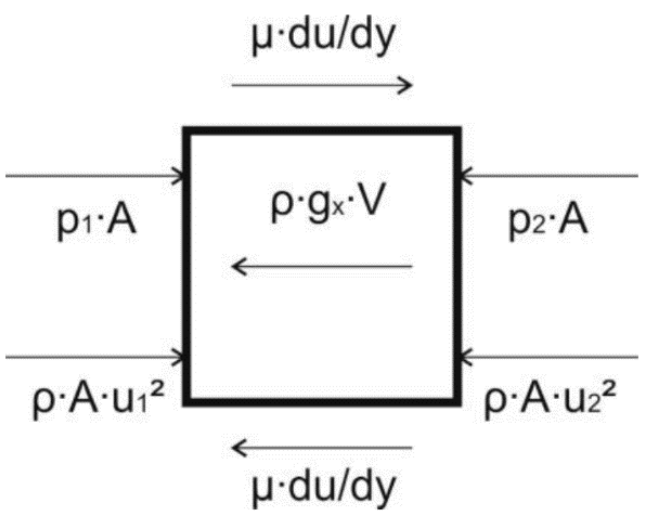

Figure 3: Forces acting on airflow

\section{$F_{p}$ from pressure differences}

Air contained in each zone has a certain pressure. When two zones of common surface A are connected by a flow model they process their pressure information $\mathrm{p}_{1}$ and $\mathrm{p}_{2}$. The flow model calculates the resulting force.

$\mathrm{F}_{\mathrm{P}}=-\mathrm{A} \cdot\left(\mathrm{p}_{\mathrm{j}}-\mathrm{p}_{\mathrm{i}}\right)$

\section{$F_{M}$ from momentum difference}

The characteristic velocity vectors of adjacent zones are processed from the zone model to the flow model. According to the flow direction $(\mathrm{x}, \mathrm{y}$ or $\mathrm{z}$ ) the flow model chooses the proper component of the velocity vectors $\left(\mathrm{u}_{1}, \mathrm{v}_{1}, \mathrm{w}_{1}\right.$ and $\left.\mathrm{u}_{2}, \mathrm{v}_{2}, \mathrm{w}_{2}\right)$ to compute the force resulting from the momentum difference between the adjacent zones taking into account the surface $\mathrm{A}$ and mean density $\rho$ of the airflow.

\begin{tabular}{cl}
\hline Direction & Momentum forces \\
\hline $\mathrm{x}$ & $\mathrm{F}_{\mathrm{M}, \mathrm{x}}=-\rho \cdot \mathrm{A} \cdot\left(\mathrm{u}_{\mathrm{j}}^{2}-\mathrm{u}_{\mathrm{i}}^{2}\right)$ \\
$\mathrm{y}$ & $\mathrm{F}_{\mathrm{M}, \mathrm{y}}=-\rho \cdot \mathrm{A} \cdot\left(\mathrm{v}_{\mathrm{j}}^{2}-\mathrm{v}_{\mathrm{i}}^{2}\right)$ \\
$\mathrm{z}$ & $\mathrm{F}_{\mathrm{M}, \mathrm{z}}=-\rho \cdot \mathrm{A} \cdot\left(\mathrm{w}_{\mathrm{j}}^{2}-\mathrm{w}_{\mathrm{i}}^{2}\right)$
\end{tabular}

Gravitational forces $F_{G}$

Gravitational forces occur in the downward direction. To compute the gravitational force, the surface A and the length $\Delta \mathrm{z}$ of the flow path are considered to calculate the volume $\mathrm{V}$ of the airflow. Multiplied with the mean density $\rho$ and the acceleration $g$ it yields a force.

$F_{G}=-\rho \cdot g \cdot A \cdot \Delta z_{i j}$

\section{Viscous forces $F_{V, \text { Flow }}$}

In the selected approach of the VEPZO model, flows are connected and exchange information with zones only. However, to calculate the shear stress, an information exchange between parallel flow models would be necessary. To avoid connections between the flow models, viscous losses are calculated in the zone models but used in the flow models. The characteristic velocity vector provided by zones enables the flow model to calculate the gradient of the two velocity components perpendicular to the flow model direction. For example, a flow model in z-direction can deliver the variation of the characteristic velocities $\mathrm{u}_{1}, \mathrm{u}_{2}$ and $\mathrm{v}_{1}, \mathrm{v}_{2}$ in the $\mathrm{x}$ and $y$-directions. If a wall is adjacent to the zone, the velocity at the wall is assumed to be zero. Therefore, the gradient is equal to the characteristic velocity divided by half the distance of the zone's centre from the wall.

The gradient information is transmitted from the flow model to the zone model. In the zone model this gradient causes shear stresses on its boundaries. This shear stress takes into account the dynamic viscosity $\mu$ and the derivation of the velocity w.r.t. the two other Cartesian directions. Summing these shear stresses along the boundaries and multiplying them with the surface area yields the viscous forces in the zones.

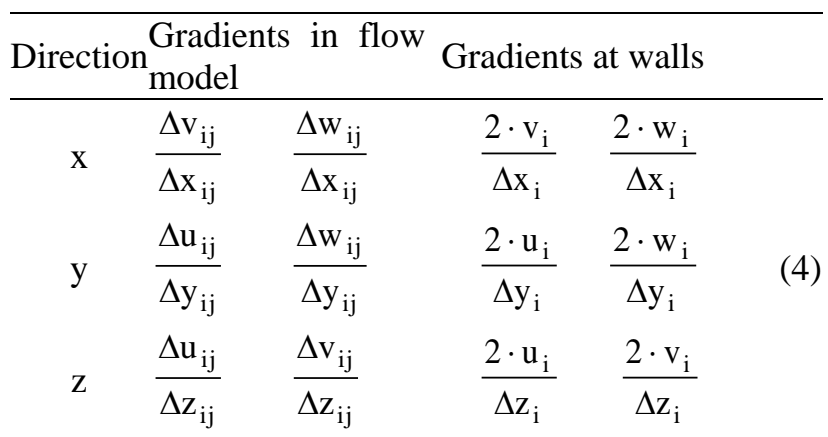

The forces acting on a flow path are summed up. This yields the acceleration of the portion of air contained in the flow path connecting two zones:

$$
A \cdot \Delta x_{12} \cdot \rho \cdot \dot{u}=F_{p}+F_{M, x}+F_{V x, F l o w}+F_{G, x}
$$

The mass flow is obtained straight forward from the velocity in a flow path. This mass flow information is transmitted to the zone model.

$$
\dot{m}_{x}=\rho \cdot A \cdot u
$$

\section{3 . Experimental Validation}

The VEPZO model was originally developed to transiently calculate the indoor climate of an aircraft cabin in a short time and to be able to develop an optimal climate for passenger comfort and temperature distribution. For such applications, the simulation was validated in (Norrefeldt et al. 2015). The experimental results used to validate the Thermal Model tests are performed in the Thermal Test Bench that has been set up in the CleanSky (Clean Sky JU 2011) project. Simulation of heated equipment were compared to measurements under various conditions which show 
that the model predicts equipment temperatures within an accuracy of 1 Kelvin.

For the model setup, the Modelica Thermal Model Generation Tool was implemented. Developed by Pathak et al. (2014) it aims to enable the user to set up a geometrically correct thermal model for complex geometries that allows predicting the impact of heated devices and their location on indoor climate.

To optimize the indoor climate, the VEPZO model has also been coupled with a genetic optimization algorithm. This algorithm optimizes a set of different solutions by combination and selection based on Darwin's theory of evolution. Using the example of a hybrid ventilated classroom (window and additional mechanical ventilation) under cold ambient conditions, an optimal use of tilt windows and the arrangement of heating devices to create optimal comfort and air quality in the occupied area was investigated (Norrefeldt et al. 2013; Reim et al. 2015).

To further validate the VEPZO model, Lindner et al. (2019) evaluated various climatic scenarios in an aircraft galley near an exterior door (Figure 4). The door represents a thermal bridge due to the structural reinforcement of the metallic frame. As a result, the crew often suffers from uncomfortably cold feet. With the help of the VEPZO model, different approaches to locally improve this area were evaluated by simulation and the most promising solutions were implemented in the test setup. Due to the short simulation time of about 10 minutes on a laptop with $2.7 \mathrm{GHz}$, many different scenarios could be evaluated in a flexible and timesaving way.

Figure 4 shows the comparison between the measured (top) and simulated (bottom) temperature map. The black dots describe sensor locations for the measurement and zone or surface centres in the simulation. The zonal grid is thus more refined than the measurement grid. As the colour maps are interpolated between the points, this geometrical difference leads to different apparent colour gradients.

Comparing the plots show that the temperatures in the left and right bottom corner are accurately predicted. Because the adjacent zone is geometrically closer than the adjacent sensors, the colour interpolation of the plot seemingly shows a larger cold zone in the measurement. In the middle of the galley, a hot air exhaust is implemented at ground level. This leads to increased surface temperature and to an increased temperature of the bottom zone, especially between positions $\mathrm{C}$ and $\mathrm{D}$. A similar effect is measured, too. The plots in this area seemingly differ because the zone above is closer than the next sensor in the vertical direction. This leads to a seemingly narrower heated zone in the measured plot than in the simulated one.

At the jumpseat in position $\mathrm{D}$, a warm mounting plume is both simulated and confirmed by measurement.
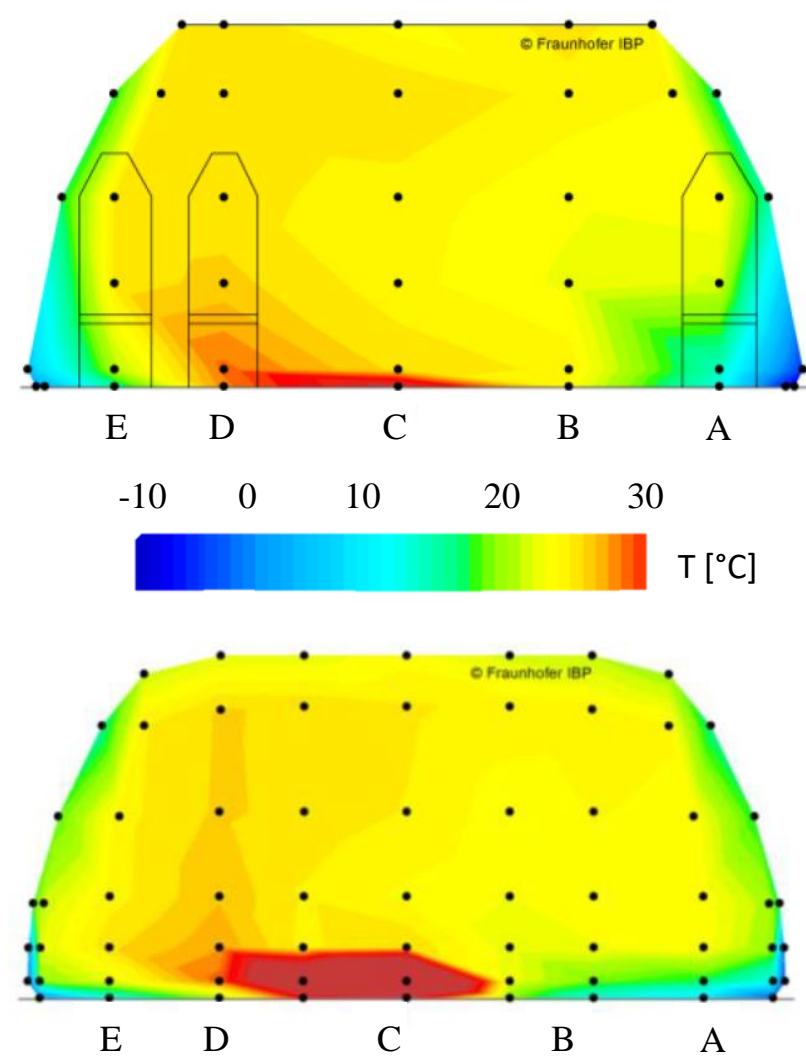

Figure 4: Experimental results of temperature distribution from derived improvement by means of an air heater (top) and results from simulation (bottom)

\section{Extension for the evaluation of the risk of infection with Covid-19}

As part of the Fraunhofer vs. Corona initiative, the VEPZO model is extended to include the spread of potentially infectious aerosols and viruses in the indoor environment. The aim is to assess potential Covid-19 infection risks in enclosed spaces. For this purpose, humans become a heat and virus source in the simulation. The viral concentration was added as an extra property to the air medium model. This results in the local distribution of the viral load emitted by an infected person in the room. The source strength is described using the concept of Quanta (Buonanno et al, 2020, Jimenez 2020) and can be adapted to the activity of the person (e.g. 2.3 quanta/h for breathing, 11.4 quanta/h for speaking and 65.1 quanta/h for loud speaking) and to personal protective equipment (none, different types of masks, etc.). The assumption is, that a higher exposure to a predicted concentration of quanta correlates with an increased infection risk.

Modelling can be used to evaluate measures to reduce the viral load. Such devices are functionally described 
in the model, for example, increased ventilation diluting the quanta concentration or air filtration removing quanta from the aspired air. Product specific performance data, which are determined in the laboratories of Fraunhofer IBP, can also be replicated in these models. Due to the current situation in the Corona pandemic, such a predictive model is a valuable tool and of great importance to be able to prevent possible transmission of infections.

A second concept to tackle the indoor hygiene in the simulation is the concept of age of air. For this, an equally distributed source of an arbitrary tracer gas is distributed in the indoor space and the relative concentrations are compared. In the zonal model, this is achieved by adding an internal source of an "extra property" to each zone. The intensity is proportional to the zone's volume. In the simulation, areas close to the air inlet will thus have a relatively lower concentration of such tracer and thus a low age of air, whereas poorly ventilated spots or zones further downstream will compute a higher concentration and thus a high age of air.

The difference between both concepts is that the viral emission considers a local source and its spread in space whereas the age of air globally identifies where relatively new respectively old air is available.

\section{Application}

A well-documented superspreading event in a restaurant in Guangzhou ( $\mathrm{Lu}$ et al. 2020) was used as an application and verification example of the modelling. Starting from an index patient, the distribution of the infectious aerosols is predicted. Figure 5 shows the considered case of the restaurant and the ventilation system. In retrospect, nine infections with Covid-19 of members of three families could be attributed to this event (Lu et al. 2020). All infected guest were seated on the left three tables. Said families were in the restaurant at the same time on January 24, 2020, together with 81 other persons ( 73 guests, 8 employees). The airflow rate was 1 litre per second and per person, divided among a total of five AC units (air conditioning). The air was only circulated, there was no fresh air supply.

The simulation of a two hour exposure with the zonal model with quanta emission took $1 \mathrm{~min}$ on a normal notebook (1.6 GHz procressor with 8GB RAM). The simulation was performed using the Esdirk 23 - order 3 stiff solver with a tolerance of 0.0001 and a fixed time step of 30s. Flags were used for sparse, parallel computation on 4 cores.

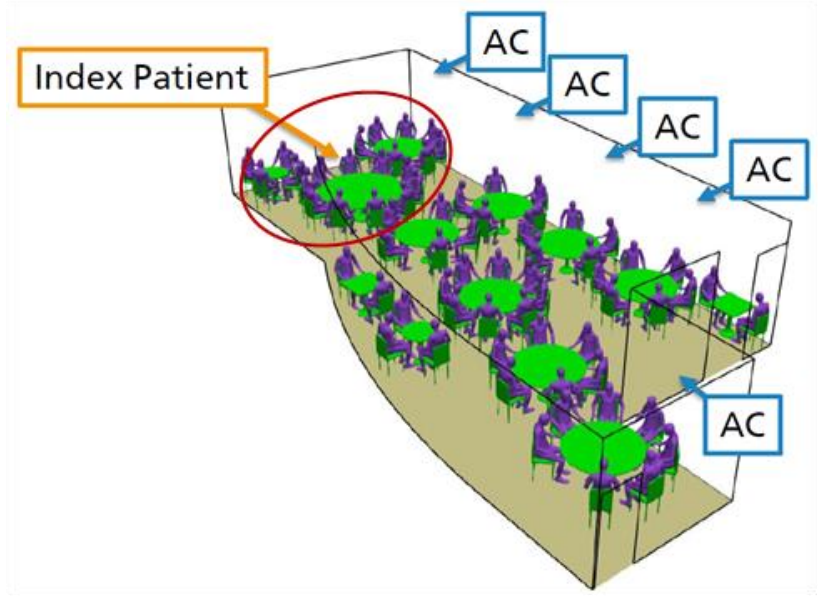

Figure 5: Schematic view of the ventilation system of the considered case and area where infetions occurred (marked in red)

Figure 6 shows the predicted age of air and the area of increased quanta load calculated using the VEPZO model. A challenge for the validation of such models is the absence of measurement data for the distribution of viral load. However, the comparison of the predicted area of increased quanta load with the documented infections shows that both are well inline.

The poor ventilation and low air exchange rate in this case played a central role, which prevented rapid renewal of the room air from taking place. This resulted in air enriched with quanta load not being removed sufficiently quickly. At the same time, it can be seen that an increased local age of air is evident along the entire length of the restaurant, but due to the airflow pattern, infection only occurred in the left area.
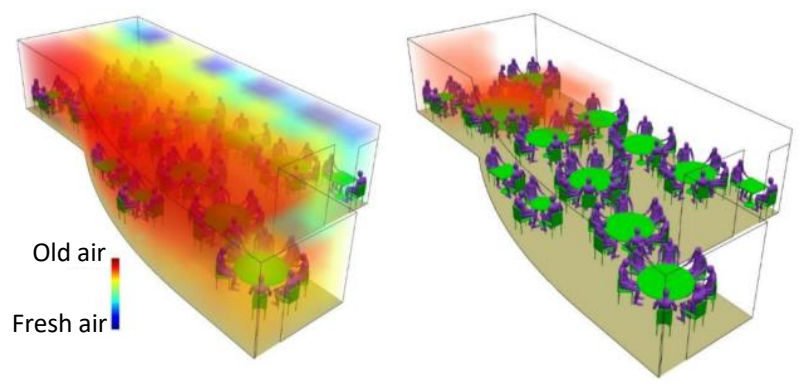

Figure 6: Simulation of the documented case: age of air (left) and increased quanta load in red (right)

With the help of the VEPZO model, an alternative ventilation pattern avoiding mixture of air was investigated to keep the dispersion area of the infectious aerosols more locally confined. Figure 7 shows the modified ventilation where fresh air is supplied centrally at the ceiling along the entire length of the restaurant and exhausted at the lower edges of the room. An improvement in the age of air and particle dispersion paths is clearly visible (Figure 8). With this ventilation 
arrangement, it is assumed that the number of infected other guests would have been lower.

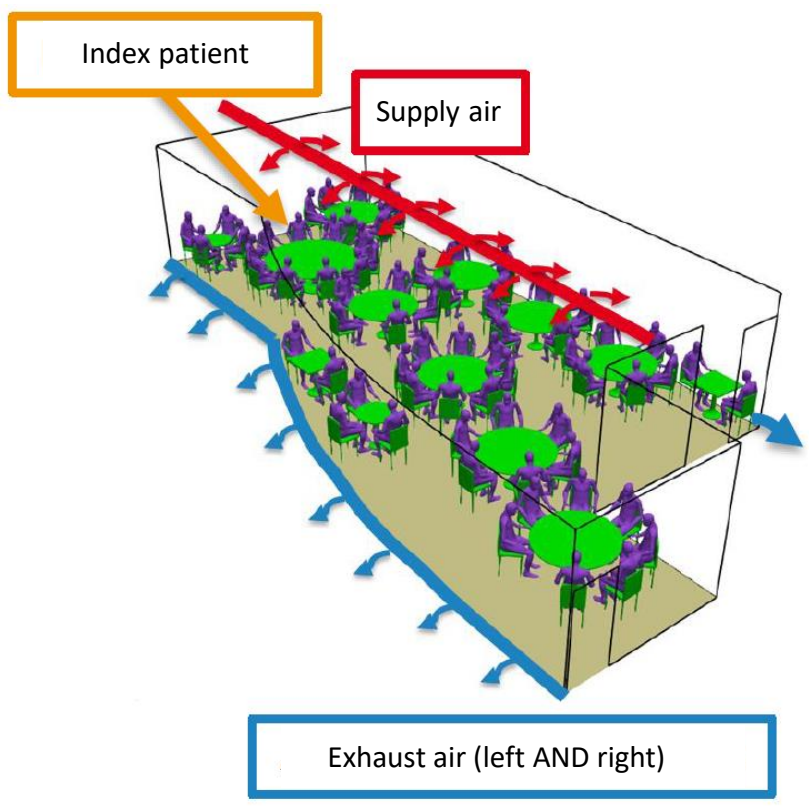

Figure 7: Schematic view of an optimum ventilation system for the considered case found with the VEPZO model
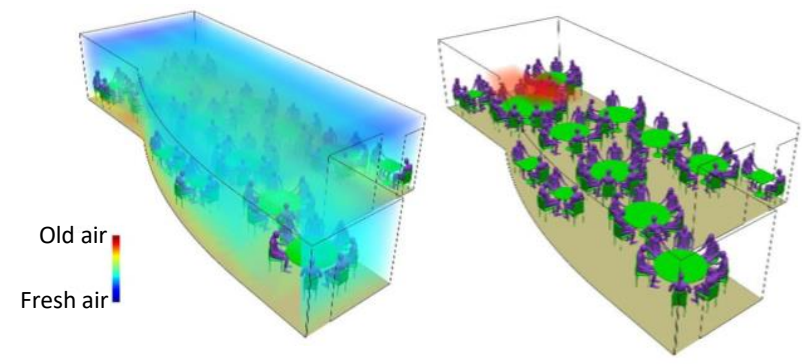

Figure 8: Simulation of the optimised case: age of air (left) and increased quanta load in red (right)

\section{Conclusion}

It is shown that the VEPZO model allows quick predictions of the local age of air and the dispersion of compounds such as virally loaded aerosols in indoor spaces. In particular, this simulation uses the notion of quanta to represent Sars-CoV-2 loaded aerosols that remain for longer time within air. Computations converge in the order of minutes on a normal PC and thus the burden to conduct such simulations is considerably lower using the zonal modelling approach than classically applied CFD methods.

For a final risk assessment, the duration of a person's stay in such a particle cloud needs to be taken into account, as well as the actual accumulation of infectious viruses in the air due to various expiratory activities of the infected person (breathing, coughing, sneezing, singing) and the vulnerability of the inhaling person.
Nevertheless, a verified method is presented by which the potential risk of infection with Covid-19 and corresponding super spreading events can be predicted by means of zonal models. Further investigations can be based on this study and improvement measures can be evaluated for their effectiveness in a short time in a product-neutral or product-specific manner.

\section{References}

Buonanno, G., Stabile, L., and Morawska, L., (2020), "Estimation of airborne viral emission: Quanta emission rate of SARS-CoV-2 for infection risk assessment," Environment International 141, doi:10.1016/j.envint.2020.105794

Clean Sky JU (2011). Available online at https://cleansky.eu/, updated on 2020, checked on $1 / 14 / 2021$

Goscé, Lara; Barton, David A. W.; Johansson, Anders (2014): Analytical modelling of the spread of disease in confined and crowded spaces. In Scientific reports 4, p. 4856 . DOI: $10.1038 / \mathrm{srep} 04856$.

Jimenez, J.L., (2020), "SARS-CoV-2 aerosol transmission estimator," https://docs.google.com/spreadsheets/d/16K1OQkLD4 BjgBdO8ePj6ytf-RpPMlJ6aXFg3PrIQBbQ/edit\#gid=519189277

Lindner, Andreas J.M.; Pschirer, Marie; Norrefeldt, Victor; Siede, Markus (2019): Case studies validating a new climate concept for cold galley areas with the DressMAN and the IESS model. In AST.

Lu, Jianyun; Gu, Jieni; Li, Kuibiao; Xu, Conghui; Su, Wenzhe; Lai, Zhisheng et al. (2020): COVID-19 Outbreak Associated with Air Conditioning in Restaurant, Guangzhou, China. In Emerging Infectious Diseases 71 (15), pp. 841-843. DOI: 10.3201/eid2607.200764

Melikov, Arsen K. (2020): COVID-19: Reduction of airborne transmission needs paradigm shift in ventilation. In Building and Environment 186, p. 107336. DOI: 10.1016/j.buildenv.2020.107336.

Norrefeldt, Victor; Grün, Gunnar; Sedlbauer, Klaus (2012): VEPZO - Velocity propagating zonal model for the estimation of the airflow pattern and temperature distribution in a confined space. In Building and Environment 48, pp. 183-194. DOI: 10.1016/j.buildenv.2011.09.007.

Norrefeldt, Victor; Grün, Gunnar; van Treeck, Christoph (2013): Use of the VEPZO model to optimize a hybrid ventilation system.

Norrefeldt, Victor; Pathak, Arnav; Lemouedda, Abdellah; Siede, Markus; Grün, Gunnar (2015): 
Validation of the zonal thermal model VEPZO/RADZO for cold outside conditions on a business jet mock-up. In AST.

Pathak, Arnav; Norrefeldt, Victor; Lemouedda, Abdellah; Grün, Gunnar (2014): The Modelica Thermal Model Generation Tool for Automated Creation of a Coupled Airflow, Radiation Model and Wall Model in Modelica. In : Proceedings of the 10th International Modelica Conference, March 10-12, 2014, Lund, Sweden. the 10th International Modelica Conference, March 10-12, 2014, Lund, Sweden, March 10-12, 2014 : Linköping University Electronic Press (Linköping Electronic Conference Proceedings), pp. 115-124.

Reim, Hanna; Norrefeldt, Victor; Noisten, Peter; Nasyrov, Vladislav; Stratbücker, Sebastian (2015): Export of BIM data to energy simulation tools and more refined zonal models. In Lake Constance 5DConference.

Sze To, G. N.; Chao, C. Y. (2010): Review and comparison between the Wells-Riley and dose-response approaches to risk assessment of infectious respiratory diseases. In Indoor Air 20.

Villafruela, J. M.; Olmedo, I.; San José, J. F. (2016): Influence of human breathing modes on airborne cross infection risk. In Building and Environment 106, pp. 340-351. DOI: 10.1016/j.buildenv.2016.07.005.

Yan, Yihuan; Li, Xiangdong; Shang, Yidan; Tu, Jiyuan (2017): Evaluation of airborne disease infection risks in an airliner cabin using the Lagrangian-based WellsRiley approach. In Building and Environment 121, pp. 79-92. DOI: 10.1016/j.buildenv.2017.05.013.

Zhang, Lei; Li, Yuguo (2012): Dispersion of coughed droplets in a fully-occupied high-speed rail cabin. In Building and Environment 47, pp. 58-66. DOI: 10.1016/j.buildenv.2011.03.015. 